\title{
Excitation of $\mathrm{Pr}^{3+}$ ions in alkaline-earth fluorides
}

\author{
E Radzhabov ${ }^{1,2}$ and V Nagirnyi ${ }^{3}$ \\ ${ }^{1}$ Vinogradov Institute of Geochemistry, Russian Academy of Sciences, Favorskii street 1a, \\ P.O.Box 4019, 664033 Irkutsk, Russia \\ ${ }^{2}$ Irkutsk State University, Physics department, Gagarin boulevard 20, 664003 Irkutsk, Russia \\ ${ }^{3}$ Institute of Physics, University of Tartu, Riia 142, 51014 Tartu, Estonia \\ E-mail: eradzh@igc.irk.ru
}

\begin{abstract}
Emission and excitation spectra of $\mathrm{CaF}_{2}, \mathrm{SrF}_{2}, \mathrm{BaF}_{2}$ doped by $\mathrm{PrF}_{3}$ were measured in vacuum ultraviolet region at $11 \mathrm{~K}$. Emission spectra of several $5 \mathrm{~d}-4 \mathrm{f}$ bands of $\mathrm{CaF}_{2}$ and $\mathrm{BaF}_{2}$ show evident vibronic structure with apparent line separated by $400 \mathrm{~cm}^{-1}$ from the main zero phonon line. No such structure was observed in $\mathrm{SrF}_{2}$-Pr. The absence of vibronic structure in $\mathrm{SrF}_{2}$ is assumed to be caused by the closeness of a local vibronic frequency to the lattice phonon one.

Excitation spectra of $4 \mathrm{f}-4 \mathrm{f}$ and $5 \mathrm{~d}-4 \mathrm{f}$ transitions are substantially different pointing on to the different excitation mechanisms of both groups of lines. The $4 \mathrm{f}-4 \mathrm{f}$ lines show an intense excitation peak adjacent to the exciton peak. This infers that excitons created near to $\operatorname{Pr}^{3+}$ ions can feed the $4 \mathrm{f}-4 \mathrm{f}$ transitions. The efficiency of excitation of the $4 \mathrm{f}-4 \mathrm{f}$ and $5 \mathrm{~d}-4 \mathrm{f}$ emission bands is quite low in the region of band-to-band transitions. An apparent peak at $19 \mathrm{eV}$ in the excitation spectrum of the $\mathrm{BaF}_{2}-\mathrm{Pr} 5 \mathrm{~d}-4 \mathrm{f}$ emission $(230 \mathrm{~nm})$ coincides well with that of the crossluminescence. No similar peaks were observed in $\mathrm{CaF}_{2}$ or $\mathrm{SrF}_{2}$ excitation spectra. We conclude that the main channel of energy transfer from the host to $\mathrm{Pr}^{3+}$ ions is realized resonantly through crossluminescence in $\mathrm{BaF}_{2}-\mathrm{Pr}$.
\end{abstract}

\section{Introduction}

Crystals doped by $\mathrm{Pr}^{3+}$ ions could be considered as perspective materials for scintillator or quantum cutting applications [1]. The mechanism of energy transfer from a host to Pr ions still remains one of the unsolved questions. The spectroscopic investigation in the vacuum ultraviolet region is one of the most powerful methods for studying the mechanisms of energy transfer.

After excitation by vacuum ultraviolet photons of $\mathrm{Pr}^{3+}$ ion doped into alkaline-earth fluorides two systems of lines are emitted. Ultraviolet bands observed in the region 215-370 $\mathrm{nm}$ belong to 5d-4f allowed interconfigurational transitions, and the sharp lines at 390-900 $\mathrm{nm}$ region belong to the $4 \mathrm{f}-4 \mathrm{f}$ parity forbidden intraconfigurational transitions. At low temperatures interconfigurational absorption, excitation and emission bands show rich fine structure due to interaction with vibrational modes $[2,3,4]$.

Excitation spectra of $\mathrm{BaF}_{2}-\mathrm{Pr}$ in vacuum ultraviolet region were investigated earlier [5]. The authors discussed the possibility of core excitation in the process of energy transfer to praseodymium in barium fluoride. The crossluminescence (or core-valence transitions) occurs from the valence band to the outmost core band where a hole was created by the absorption of a vacuum ultraviolet photon. The crossluminescence of undoped $\mathrm{BaF}_{2}$ consists of the main band at $220 \mathrm{~nm}$, overlapping strongly with the $\operatorname{Pr}^{3+} 4 \mathrm{f}-5 \mathrm{~d}$ absorption, and a lower-intensity band at 
$193 \mathrm{~nm}$ [6]. No core-valence transitions (or crossluminescence) were observed in $\mathrm{SrF}_{2}$ or in $\mathrm{CaF}_{2}$ where these transitions are energetically unfavourable $[6]$.

The main goal of this paper is the clarification of $\operatorname{Pr}^{3+}$ emission and excitation mechanisms by comparative spectroscopic study of the three homologous hosts $\mathrm{CaF}_{2}, \mathrm{SrF}_{2}, \mathrm{BaF}_{2}$ with different concentration of $\mathrm{PrF}_{3}$ dopants in vacuum ultraviolet region.

\section{Experimental}

Crystals were grown in vacuum in a graphite crucible by the Stockbarger method. Graphite crucible contains three cylindrical cavities $10 \mathrm{~mm}$ in diameter and $80 \mathrm{~mm}$ long, which allows to grow three crystals of $\varnothing 10 \times 50 \mathrm{~mm}$ in dimensions with different impurity concentrations at the same time. As the first step a large oxygen-free crystal was grown with addition of $\mathrm{CdF}_{2}$ as an oxygen scavenger in a simple large volume crucible. Then parts of this crystal were used for growing the doped crystals.

Emission and excitation spectra as well as the emission decay kinetics of $\mathrm{CaF}_{2}, \mathrm{SrF}_{2}$ and $\mathrm{BaF}_{2}$ doped by 0.15 mol. \% of $\mathrm{PrF}_{3}$ were studied in the region of $2-24 \mathrm{eV}$ at $11 \mathrm{~K}$. The measurements were conducted at the SUPERLUMI station of HASYLAB at DESY (Hamburg, Germany) [7]. Additionally some emission spectra were measured at the Institute of Geochemistry using $\mathrm{Kr}$ $(120 \mathrm{~nm})$ or Xe $(147 \mathrm{~nm})$ discharge lamps as excitation sources and a solar-blinde photomultiplier FEU142 attached to a vacuum grating monochromator VM4.

\section{Results}

The emission spectra due to the $5 \mathrm{~d}-4 \mathrm{f} \operatorname{Pr}^{3+}$ transitions are shown on figure (Fig.1). The observed emission lines are due to transitions from the lowest 5 d-level to different $4 \mathrm{f}$ levels of the $\mathrm{Pr}^{3+}$ ion, whose energies in $\mathrm{CaF}_{2}$ are known from [8]. Five 5d levels of $\mathrm{Pr}^{3+}$ ion are split by the crystal field in two groups. Usually the $5 \mathrm{~d}-4 \mathrm{f}$ emission lines are wider than the $4 \mathrm{f}-4 \mathrm{f}$ lines due to the fact that the outmost $\mathrm{d}$ - levels interact with lattice vibration stronger than the inner $\mathrm{f}$ - levels. The $5 \mathrm{~d}-4 \mathrm{f}$ emission spectrum of $\mathrm{CaF}_{2}-\mathrm{Pr}$ is similar to those measured earlier [3]. Each emission band of $\mathrm{CaF}_{2}$ and $\mathrm{BaF}_{2}$ shows a prominent zero phonon line and another strong line of smaller intensity separated by near $400 \mathrm{~cm}^{-1}$. From additional measurements of lower doped crystals the fine structure of $5 \mathrm{~d}-4 \mathrm{f}$ emission spectra was evidently observed at $78 \mathrm{~K}$ in $\mathrm{BaF}_{2}-0.01 \% \mathrm{PrF}_{3}$, the structure is less evident in $\mathrm{CaF}_{2}-0.01 \% \mathrm{PrF}_{3}$ and fully absent in $\mathrm{SrF}_{2}-0.01 \% \mathrm{PrF}_{3}$.

The shape of emission spectra are compared in one plot by shifting the spectra by 44300 , $45300,45500 \mathrm{~cm}^{-1}$ for $\mathrm{CaF}_{2}, \mathrm{SrF}_{2}, \mathrm{BaF}_{2}$, respectively (Fig.2). The spectra of $\mathrm{CaF}_{2}$ and $\mathrm{BaF}_{2}$ are similar, while the separation between the sharp lines is slightly smaller in $\mathrm{BaF}_{2}$. The spectrum of $\mathrm{SrF}_{2}$ is structureless.

Excitation spectra for the 5d-4f (for the line $5 \mathrm{~d}-{ }^{3} \mathrm{H}_{4}$ ), 4f-4f (for the line ${ }^{3} \mathrm{P}_{0}-{ }^{3} \mathrm{H}_{4}$ ) and exciton emissions are shown in Figure 3. The excitation curves of the $5 \mathrm{~d}-4 \mathrm{f}$ and $4 \mathrm{f}-4 \mathrm{f}$ emission are different in the region of direct 5d-4f excitation 5.5-9 eV. Prominent excitation bands were observed below the exciton peaks only. Both spectra are of low intensity and very smooth above the exciton peaks in the range of interband transition (see Fig.3).

4f-4f excitation The excitation spectra of the ${ }^{3} \mathrm{P}_{0}-{ }^{3} \mathrm{H}_{4}$ emission line show the peaks near $7 \mathrm{eV}$ (see Fig.3). This band is most intense in $\mathrm{CaF}_{2}$ less pronounced in $\mathrm{SrF}_{2}$ and the weakest in $\mathrm{BaF}_{2}$ crystals. It belongs to the aggregate Pr defects, which possess photon cascade emission [9]. All three crystals show intensive excitation bands right below the exciton peak. Similarly situated wide peaks were observed earlier for the $4 \mathrm{f}-4 \mathrm{f}$ emission of several rare- earth ions in $\mathrm{LaF}_{3}[10]$.

5d-4f excitation The excitation spectra of the $5 \mathrm{~d}-4 \mathrm{f}$ emissions in $\mathrm{CaF}_{2}$ and $\mathrm{SrF}_{2}$ show a sharp decrease at the exciton edge energies (see Fig.3). The decrease is less evident in $\mathrm{BaF}_{2}$. The spectra are of low intensity and almost structureless in the region of the interband transitions. The prominent excitation peak near $19.5 \mathrm{eV}$ was observed for $\mathrm{BaF}_{2}$, it was absent in $\mathrm{CaF}_{2}$ and 

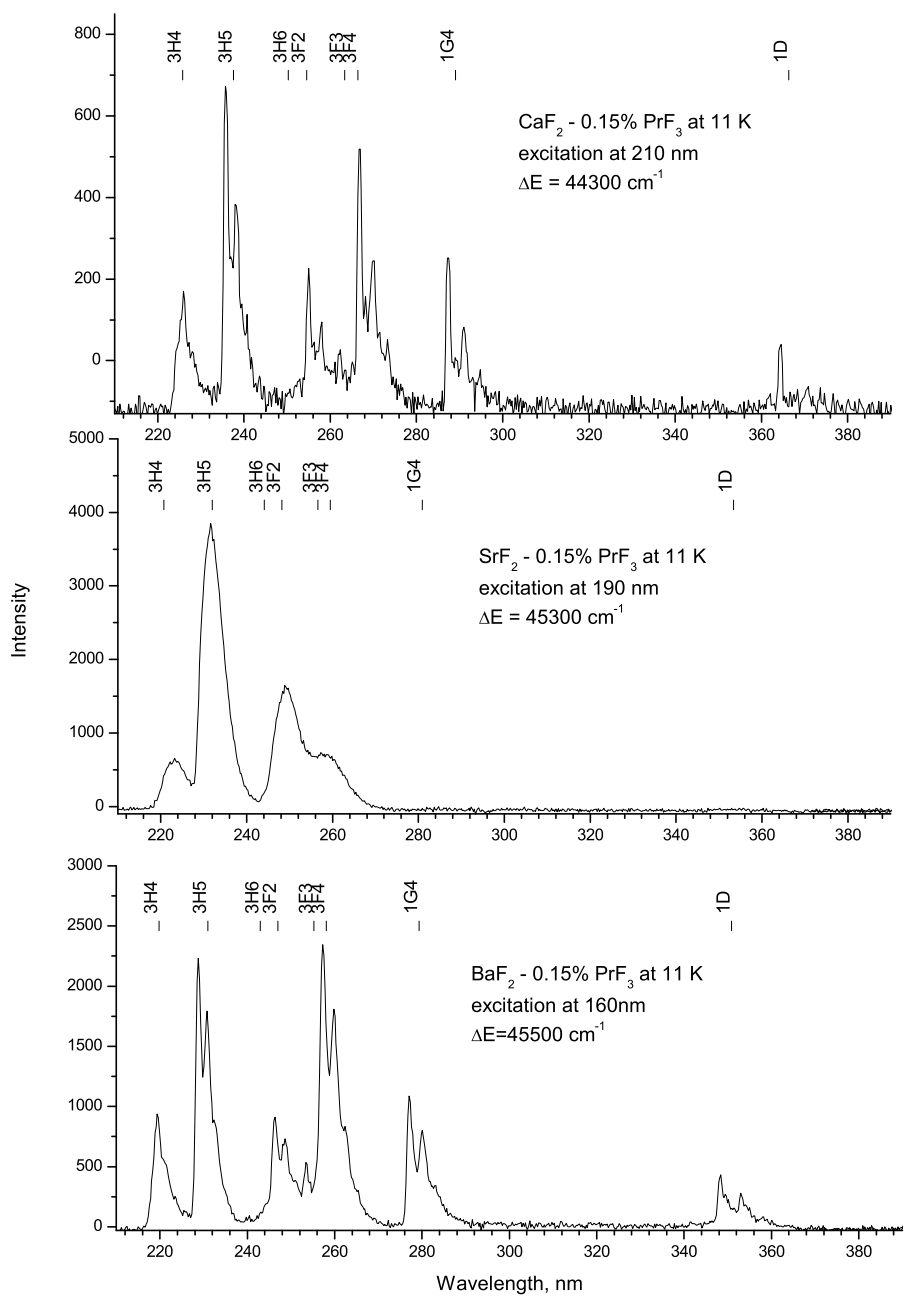

Figure 1. Emission spectra of $\mathrm{Pr}^{3+} 5 \mathrm{~d}-4 \mathrm{f}$ transitions in $\mathrm{CaF}_{2}, \quad \mathrm{SrF}_{2}, \mathrm{BaF}_{2}$ crystals doped by 0.15 molar \% of $\mathrm{PrF}_{3}$. Positions of each line due to the transitions from the lowest $5 \mathrm{~d}$ levels to individual if levels are shown at the top of each plot.

$\mathrm{SrF}_{2}$ crystals. The excitation efficiency at $19.5 \mathrm{eV}$ is only twice as low as that in the region of a direct 4f-5d excitation (see Fig.3).

To verify the possibility of energy transfer from the host to a praseodymium ion due to the absorption of a crossluminescence photon by $5 \mathrm{~d}-4 \mathrm{f}$ states we compare the emission spectra of undoped and $\mathrm{Pr}$-doped $\mathrm{BaF}_{2}$ with the absorption spectrum of $\mathrm{Pr}^{3+}$ ion in $\mathrm{BaF}_{2}$ (Fig.4). The absorption is the highest at 200-220 $\mathrm{nm}$ where it largely overlaps with $220 \mathrm{~nm}$ crossluminescent bands (see Fig.4). With increasing Pr concentration the intensity of the Pr emission lines grows, while the intensity at $210 \mathrm{~nm}$, which is mostly due to the crossluminescence, is continuously decreased partially due to the increasing Pr absorption. The changes in crossluminescence intensity could be monitored via the intensity of the $193 \mathrm{~nm}$ band, where the Pr absorption is relatively low. The intensity of the $193 \mathrm{~nm}$ band also decreases with increasing Pr concentration (see Fig.4).

\section{Discussion}

It should be noted that fine structure of spectra was not observed in $\mathrm{SrF}_{2}-0.15 \% \mathrm{PrF}_{3}$ crystal (see Fig.1). Usually many physical properties changes continuously in the row $\mathrm{CaF}_{2}-\mathrm{SrF}_{2}-\mathrm{BaF}_{2}$ [11]. Therefore the observed dependence is very unusual. The transitions from the $5 \mathrm{~d}$ to $4 \mathrm{f}$ state 


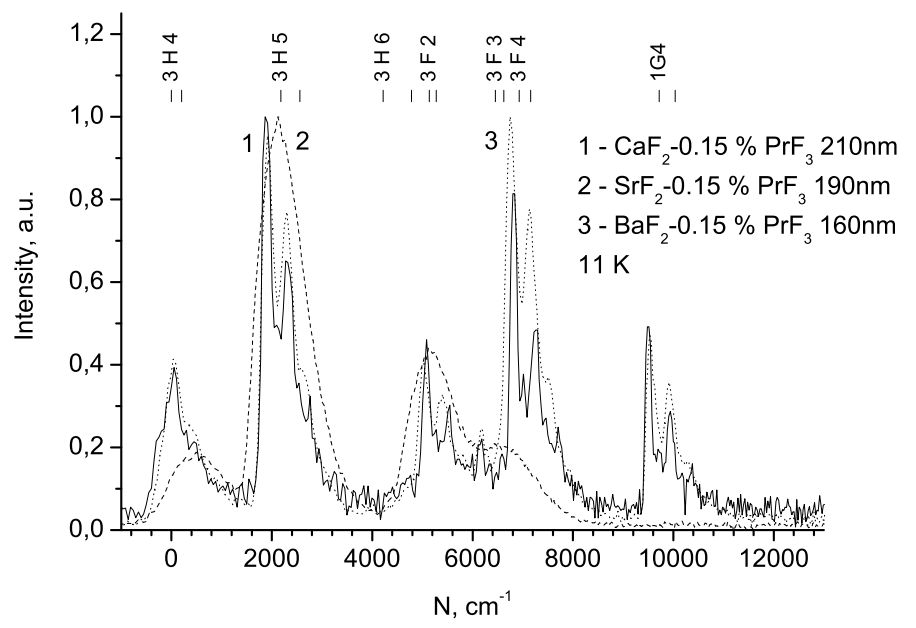

Figure 2. Comparison of the shapes of the emission spectra of $\mathrm{Pr}^{3+} 5 \mathrm{~d}-4 \mathrm{f}$ transitions in $\mathrm{CaF}_{2}, \quad \mathrm{SrF}_{2}, \quad \mathrm{BaF}_{2}$. The spectra are normalized for the ${ }^{3} \mathrm{H}_{5}$ line intensities, while the origin of the wave number scale is placed at the ${ }^{3} \mathrm{H}_{4}$ line.

are often assisted by lattice phonons. As a result the optical emission or absorption spectrum

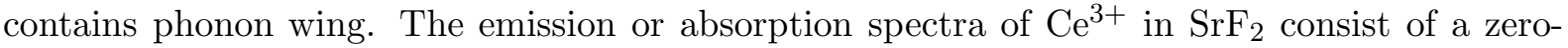
phonon line and a broad phonon wing shifted from line by $400 \mathrm{~cm}^{-1}$ [12]. The frequencies of a longitudinal optic phonon obtained from optical data at $5 \mathrm{~K}$ are 484,397 and $346^{-1}$ for $\mathrm{CaF}_{2}$, $\mathrm{SrF}_{2}, \mathrm{BaF}_{2}$ respectively [11]. Typical distances between the vibronic lines in absorption spectra were $450,430,400 \mathrm{~cm}^{-1}$ for the $0.005 \% \mathrm{PrF}_{3}$ doped $\mathrm{CaF}_{2}, \mathrm{SrF}_{2}, \mathrm{BaF}_{2}$ crystals respectively [4]. The spectrum of several individual Pr emission lines caused by transitions from the $5 \mathrm{~d}$ to ${ }^{3} \mathrm{H}_{4},{ }^{3} \mathrm{~F}_{2},{ }^{3} \mathrm{~F}_{3},{ }^{1} \mathrm{G}$ states in $\mathrm{BaF}_{2}$ and $\mathrm{CaF}_{2}$ is shown in Fig.2. Besides the zero-phonon lines the intensive vibrational lines separated by 430 and $390 \mathrm{~cm}-1$ were observed in $\mathrm{CaF}_{2}$ and $\mathrm{BaF}_{2}$, respectively. For $\mathrm{CaF}_{2}$ similar $5 \mathrm{~d}-4 \mathrm{f}$ emission spectra of $\mathrm{Pr}$ ion were measured with higher resolution earlier [3]. Obviously these phonon lines are due to a local vibration in the complex $\mathrm{PrF}_{8}$ (see also [4]). The frequencies of these local vibrations are higher than the lattice phonon frequencies in $\mathrm{BaF}_{2}$, lower then those in $\mathrm{CaF}_{2}$ and should be almost equal to the phonon frequencies in $\mathrm{SrF}_{2}$. Therefore the longitudinal optic phonons possessing the energy equal to that of the local vibrations of $\mathrm{PrF}_{8}$ should wide the Pr emission lines in $\mathrm{SrF}_{2}$ (see Fig.2). In $\mathrm{CaF}_{2}$ or $\mathrm{BaF}_{2}$, the frequencies of lattice phonons and $\mathrm{PrF}_{8}$ local vibrations are sufficiently different and therefore the structure of emission spectra can be observed.

Excitation spectra of the intraconfigurational $4 \mathrm{f}-4 \mathrm{f}$ transitions of trivalent $\mathrm{Nd}, \mathrm{Er}, \mathrm{Tm}, \mathrm{Pr}$ ions doped into several trifluorides were measured in an early paper [10]. Excitation bands on the low-energy wing of exciton bands were observed in all cases. Evidently the excitonic mechanism of the energy transfer to the f-shell has a common character. One can assume that the energy of self-trapped exciton (near $4.5 \mathrm{eV}$ in fluorides) is transfered to the appropriated excited f-levels of rare-earth ions. The exciton energy is not sufficient for the excitation of $4 \mathrm{f}-5 \mathrm{~d}$ transitions for all the rare-earth ions except $\mathrm{Ce}^{3+}$.

Excitation spectrum for the $255 \mathrm{~nm} \mathrm{Pr}^{3+}$ emission (transition $5 \mathrm{~d}^{3}{ }^{3} \mathrm{~F}_{j}$ ) was measured for $\mathrm{BaF}_{2^{-}}$ $0.3 \% \mathrm{PrF}_{3}$ earlier [5]. The integrated intensity and the fast component of the $5 \mathrm{~d}$ luminescence show a rather smooth increase in intensity above $18 \mathrm{eV}$, that is in the region of excitation of the $5 \mathrm{p}$ Ba core states. Authors assume that the energy of the core cation excitations to be transfered to Pr luminescence centres in $\mathrm{BaF}_{2}$ [5]. Our measurements show an evident Pr 5d-4f excitation peak which well coincides with the crossluminescence excitation peak in undoped $\mathrm{BaF}_{2}$ crystals. The decrease in the crossluminescence intensity accompanying the increase of Pr concentration is clearly seen from comparison of the emission intensity at the wavelength $193 \mathrm{~nm}$, where the 


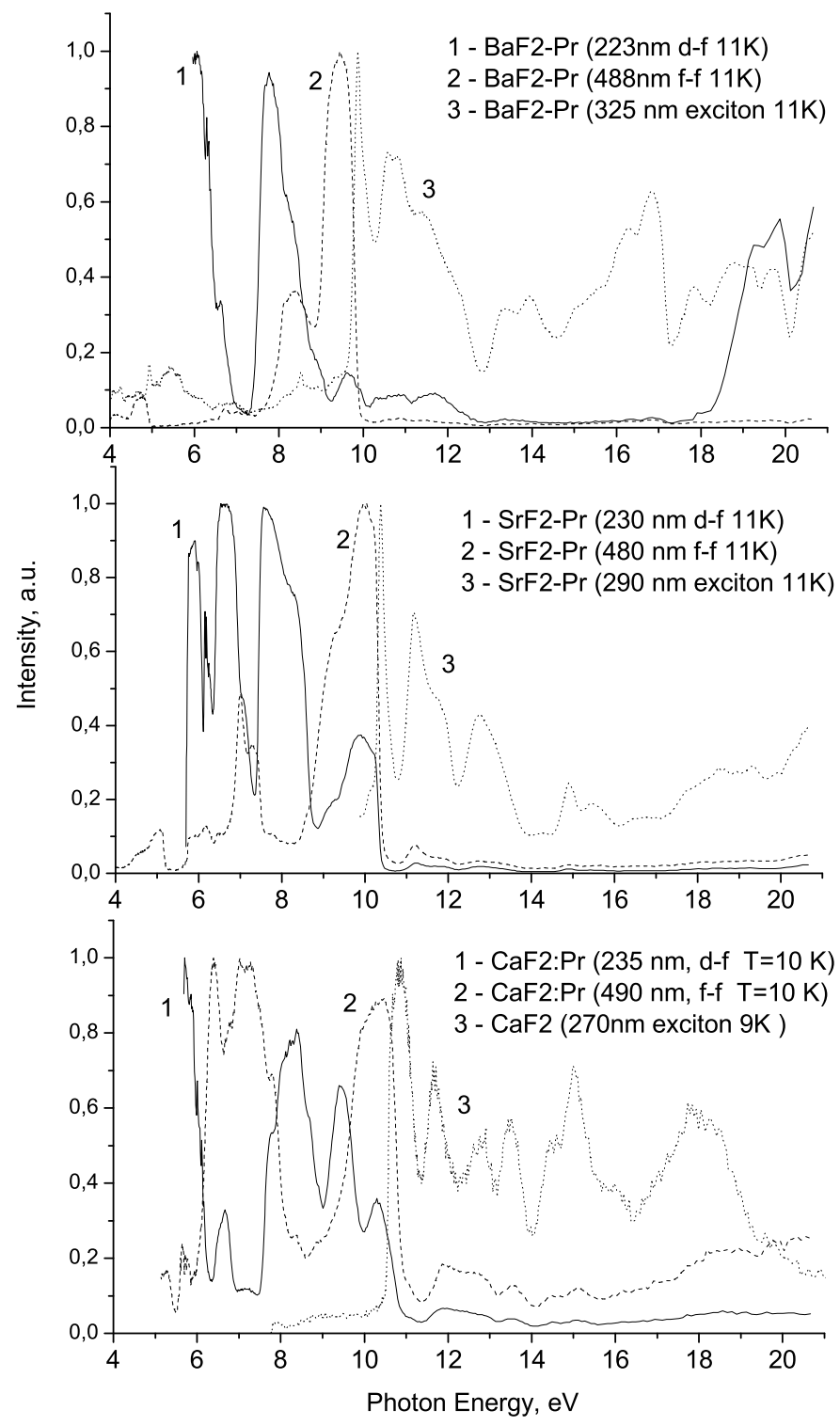

Figure 3. Excitation spectra of Pr 5d-4f and 4f-4f emission bands as well as exciton emission of $\mathrm{CaF}_{2}, \mathrm{SrF}_{2}, \mathrm{BaF}_{2}$ crystals doped by 0.15 molar $\%$ of $\mathrm{PrF}_{3}$.

Pr absorption is low (see Fig.4). At the level of 0.3 molar $\%$ of $\mathrm{PrF}_{3}$ the crossluminescence is almost completely suppressed. Considering the uniform Pr distribution across the $\mathrm{BaF}_{2}$ lattice one could estimate the radius of the resonant transfer from a crossluminescent centre to a $\mathrm{Pr}$ ion, at which the crossluminescence becomes fully suppressed, as half the distance between $\mathrm{Pr}$ ions. The half Pr-Pr distance at the level of 0.3 molar \% is near $21.5 \AA$.

One could estimate this radius also from emission and absorption spectra overlapping. The radius $R_{c}$ of dipole-dipole energy transfer defined as the distance at which the probability of donor (crossluminescent centre in our case) radiative transitions is equal to the probability of transfer to acceptor (Pr ion), is given by [13]:

$$
R_{c}^{6}=\frac{B}{n^{4} N_{A}} \int_{0}^{\infty} \frac{f_{D}(E) \mu_{A}(E)}{E^{4}} d E
$$




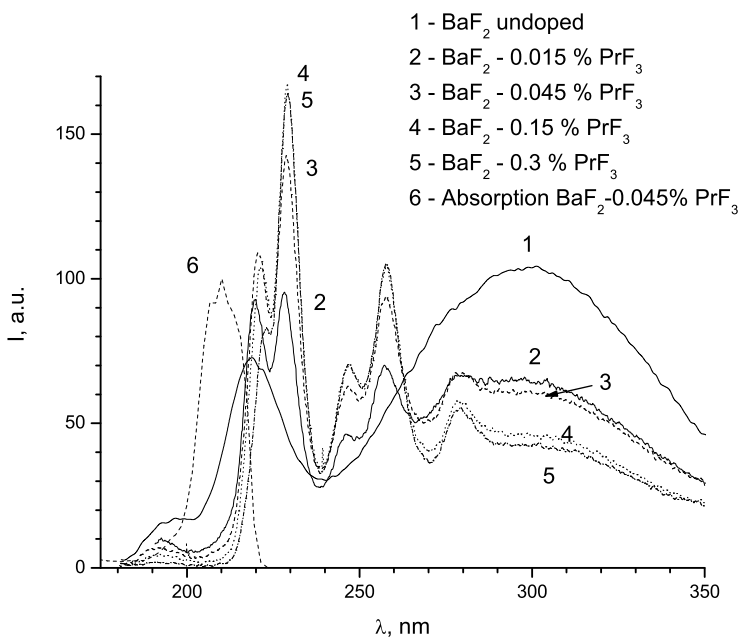

Figure 4. Emission spectra of undoped $\mathrm{BaF}_{2}$ (curve 1) and $\mathrm{BaF}_{2}-\mathrm{PrF}_{3}$ (curves 2-5) measured under x-ray excitation at room temperature. Absorption spectrum of $\mathrm{BaF}_{2}-0.045 \% \mathrm{PrF}_{3}$ in arbitrary units is added for comparison (curve 6).

Here $\mathrm{n}$ is the refractive index of crystal, the subscript A denotes the acceptor centre and D denotes the donor centre. The term $N_{A}$ is concentration of acceptor centres (in $\mathrm{cm}^{-3}$ ) and $\mu_{A}$ (E) is their absorption coefficient (in $\mathrm{cm}^{-1}$ ). The $f_{D}(E)$ is the emission spectrum of the donor centres, normalised in such a way that $\int_{0}^{\infty} f_{D}(E) d E=1$. Constant $B=\frac{3 \hbar^{4} c^{4}}{4 \pi}=$ $3.7 * 10^{-20}\left(\mathrm{eV}^{4} * \mathrm{~cm}^{4}\right)$. From the experimental data we evaluate the radius $\mathrm{R}_{c}$ as $18.3 \AA$. Thus, both values obtained for the distance of energy transfer from the host to the $\mathrm{Pr}^{3+}$ ion via the crossluminescence are in good agrement.

We can summarize the results supporting the energy transfer to the $\mathrm{Pr}^{3+}$ ions by crossluminescence in $\mathrm{BaF}_{2}$-Pr as follows:

- sufficient overlap between the crossluminecence and Pr absorption,

- continuous decrease of crossluminescence intensity (at $193 \mathrm{~nm}$ ) with Pr concentration increasing,

- appearance of the crossluminescence excitation peak near $19 \mathrm{eV}$ in the Pr 5d-4f excitation spectrum.

\section{Conclusion}

The above-described experimental results prove that the $\mathrm{Pr}^{3+} 5 \mathrm{~d}-4 \mathrm{f}$ emission is efficiently excited via the resonant energy transfer from crossluminescence centres in $\mathrm{BaF}_{2}$. The excitation mechanism in $\mathrm{CaF}_{2}$ and $\mathrm{SrF}_{2}$ should be investigated additionally.

\section{Acknowledgments}

The authors gratefully acknowledge V. Kozlovskii for growing the crystals investigated in this work. Financial support by the Estonian Science Foundation (grant 7825) and the European CommunityResearch Infrastructure Action under the FP6 Structuring the European Research Area Programme (through the Integrated Infrastructure Initiative Integrating Activity on Synchrotron and Free Electron Laser Science) is gratefully acknowledged.

\section{References}

[1] Melcher C L 2005 Nucl. Instrum. Meth. A 5376

[2] Makhov V N, Khaidukov N M, Lo D, Kirm M and Zimmerer G 2003 J. Lumin. 102-103 638

[3] Oskam K D, Houtepen A J and Meijerink A 2002 J. Lumin. 97107 
[4] Loh E, Blazey K W, Nosenzo L and Reguzzoni E 1979 J. Phys. C: Solid State Phys. 123879

[5] Rodnyi P A, Stryganyuk G B, van Eijk C W E et.al. 2005 Phys. Rev. B 72195112

[6] Rodnyi P A 1992 Sov. Phys. Solid State 341053

[7] Kirm M, Lushchik A, Lushchik C, Nepomnyashikh A I and Savikhin F 2001 Rad. Measurements 33515

[8] Hargreaves W A 1972 Phys. Rev. B 63417

[9] Radzhabov E 2009 J. Lumin. 1291581

[10] Yang K H, DeLuca J A 1978 Phys. Rev. B 174246

[11] Crystal with fluorite structure, ed by Hayes W Clarendon Press, Oxford, 1974

[12] Radzhabov E and Kurobori T 2004 J. Phys.: Cond. Matter 161871

[13] Visser R, Dorenbos P, van Eijk C W E, Meijerink A, Blasse G and den Hartog H W 1993 J.Phys. Cond. Matter $\mathbf{5} 1659$ 